Chapter 7

\title{
Neoadjuvant Chemotherapy in the Management of Advanced Ovarian Cancer and Primary Cancer of the Peritoneum: Rationale and Results
}

\author{
Maurie Markman \\ Additional information is available at the end of the chapter \\ http://dx.doi.org/10.5772/55394
}

\section{Introduction}

For more than 40 years the standard-of-care in the management of advanced ovarian cancer and primary peritoneal cancer has been an attempt to optimally cytoreduce ("debulk") disease present within abdominal cavity prior to the administration of cytotoxic chemotherapy [1-3]. Several justifications have been advanced in support of this general approach to disease management (briefly summarized in Table 1).

\footnotetext{
Remove all or most macroscopic tumor to permit the subsequent delivery (via capillary flow or intraperitoneal drug delivery) of optimal concentrations to any residual cancer of an effective anti-neoplastic chemotherapy regimen (platinum-based)
}

Reduce the risk for the development of resistance resulting from the delivery of inadequate concentrations of cytotoxic drug therapy

Rapidly decrease cancer-related symptoms due to the presence of intra-abdominal cancer (e.g., pain, inability to eat)

Enhance the nutritional status to permit improved tolerance of the chemotherapy regimen

Optimize the functional status of the immune system following removal of large tumor bulk

Table 1. Rationale for primary surgical cytoreduction in advanced ovarian cancer

A rather substantial number of single investigators, single institutions, and multi-institutional experiences have reported data that support the concept that patients who undergo successful primary surgery experience an anticipated statistically-significant superior survival outcome 
compared to patients who are treated with primary chemotherapy (no attempt to cytoreduce the cancer following the diagnosis) or who fail to be able to undergo successful cytoreduction despite a reasonable attempt by a gynecologic cancer surgeon [2-4]. Unfortunately, and rather remarkably considering the extensive use of this strategy, the utility of primary surgical cytoreduction has never actually been documented in a prospective phase 3 randomized trial.

In fact, while there should be no legitimate disagreement with the statement that the population of advanced epithelial ovarian cancer patients who initiate platinum-based chemotherapy with the smallest volume of residual cancer will be anticipated to experience the most favorable survival outcome, it remains uncertain today if the "favorable outcome" results from the skills of the individual gynecologic cancer surgeon or is more related to the "favorable biology" of the cancer which also grows and progresses in a pattern and location that is more conducive for resection, in contrast to more aggressive and widespread malignancies. For example, it is quite plausible that the same poorly understood biological/molecular factors that render a particular tumor more chemo-resistant (either de novo or early in the clinical course) also substantially influence resectability (e.g., presence or absence of diffuse carcinomatosis within the peritoneal cavity or extensive retroperitoneal involvement with the cancer).

\section{Evidence-based data supporting a role for surgical cytoreduction in advanced ovarian cancer at some point during the course of the illness}

Despite the absence of phase 3 randomized trial data demonstrating the utility of primary surgical cytoreduction in advanced ovarian cancer, a landmark European phase 3 study revealed the benefits of an interval surgical cytoreductive procedure following an initial surgery and the administration of several cycles of cytotoxic chemotherapy [5]. In this trial patients were randomized to undergo primary surgical cytoreduction followed by six cycles of platinumbased chemotherapy versus having the surgery, followed by three cycles of the same chemotherapy, which was followed by a second attempt at surgical cytoreduction ("interval cytoreduction") and then three more cycles of chemotherapy. Patients randomized to the interval surgical procedure experienced a statistically significant improvement in overall survival compared to women not offered this second surgical procedure.

However, a second study conducted in the United States which employed a similar "interval cytoreduction" approach but utilized a cisplatin plus paclitaxel regimen versus cisplatin plus cyclophosphamide utilized in the European study failed to demonstrate an improvement in survival associated with the second surgery [6]. While it is always difficult to compare the outcome results across two independent clinical trials, the difference in the overall aggressiveness of the primary surgical approach in the United States may explain (at least in part) the somewhat surprising differences between the two studies.

Considering the results of these two well-performed evidence-based studies one might suggest the data support the concept that ovarian cancer patients appear to experience benefit from an attempt to maximally cytoreduce intraperitoneal disease but it does not make a major differ- 
ence if that surgery is performed at the initial diagnosis or as a component of an attempt to administer primary chemotherapy followed by definitive surgery.

\section{Neoadjuvant chemotherapy of advanced ovarian cancer}

The rational supporting the use of neoadjuvant chemotherapy in the management of advanced ovarian cancer is outlined in Table 2. The earliest experience with this approach focused on patients who were simply too ill to undergo primary surgery, or were believed to be an unacceptable surgical risk (e.g., large bilateral cancer-related pleural effusions) [7].

Manage patients unable to undergo primary surgical cytoreduction either because of extensive disease or the existence of co-morbidities increasing the risk associated with the procedure

Reduce the morbidity and possibly mortality associated with aggressive surgery of extensive intra-peritoneal ovarian cancer

Improve the nutritional status of patients prior to the performance of a major abdominal surgery, specifically to reduce the risk of a serious adverse event.

Determine if an individual cancer is chemo-sensitive and whether it is appropriate to attempt to cytoreduce the cancer. (The basic argument here is that patients with chemo-resistant cancers are most unlikely to benefit from an attempt at surgical cytoreduction. Of course, surgery may be clinically indicated to provide short-term palliation of distressing cancer-related symptoms [e.g., colostomy for large-bowel obstruction].)

Table 2. Rationale for neoadjuvant chemotherapy of advanced ovarian cancer

However, many patients who achieved a major objective and subjective response following several cycles of platinum-based chemotherapy were found to have sufficiently improved their overall performance status to permit surgical intervention. In several reports documenting the outcome of patients managed in this manner there was evidence that the survival outcomes were not terribly different "compared to" individuals of a similar age and initial tumor volume who were managed with primary surgery followed by chemotherapy [8-10]. It is critical to acknowledge here that such comparisons are fraught with great danger due to the potential for major selection bias in the populations of individuals who might undergo neoadjuvant chemotherapy versus primary surgical cytoreduction [7].

\section{Phase 3 trial of primary surgical cytoreduction compared to neoadjuvant chemotherapy followed by surgical cytoreduction in advanced ovarian cancer}

In fact, the only way to directly address the issue of the overall clinical utility of primary surgical cytoreduction versus neoadjuvant chemotherapy and a subsequent attempt to achieve 
maximal surgical cytoreduction would be to perform a randomized trial. In another landmark clinical study a multi-institutional cooperative group consortium reported the results of a welldesigned and conducted phase 3 trial involving 670 women with advanced ovarian cancer or primary peritoneal cancer who either underwent primary surgery followed by chemotherapy with carboplatin plus paclitaxel or were treated initially (after the diagnosis was confirmed) with the same chemotherapy followed by cytoreductive surgery with additional chemotherapy subsequently administered [11].

The study outcomes are summarized in Table 3. There was no difference in survival between the two approaches, but overall serious morbidity was considerably lower in the neoadjuvant chemotherapy group. Of interest, within each country where this study was conducted it was observed that compared to the percentage of patients able to be optimally cytoreduced in the primary setting there was a higher proportion of women able to achieve this clinical state in the trial arm where neoadjuvant chemotherapy was followed by interval cytoreduction.

\begin{tabular}{lccc}
\hline & $\begin{array}{c}\text { Primary Surgical } \\
\text { Cytoreduction }\end{array}$ & $\begin{array}{c}\text { Neoadjuvant } \\
\text { Chemotherapy }\end{array}$ & Hazard ratio: 1.01 \\
\hline $\begin{array}{l}\text { Progression-Free Survival } \\
\text { (median) }\end{array}$ & 12 months & 12 months & Hazard ratio: 0.98 \\
\hline Overall Survival (median) & 29 months & 30 months & $0.7 \%$ \\
\hline $\begin{array}{l}\text { Mortality (death within 28 } \\
\text { days after surgery) }\end{array}$ & $2.5 \%$ & $4.1 \%$ & $1.7 \%$ \\
\hline Grade 3-4 hemorrhage & $7.4 \%$ & $0 \%$ & \\
\hline Infection & $8.1 \%$ & $2.6 \%$ & \\
\hline Venous complications & & & \\
\hline
\end{tabular}

Table 3. Summary of results of the phase 3 trial comparing primary cytoreductive surgery to neoadjuvant chemotherapy followed by surgery in advanced ovarian cancer ${ }^{11}$

\section{Summary and reasonable conclusions regarding utility of neoadjuvant chemotherapy of advanced ovarian cancer based on available evidence- based data}

Despite the results of this well-conducted multi-center phase 3 randomized trial there continues to be considerable controversy within the gynecologic cancer clinical research community regarding a standard/routine role for neoadjuvant chemotherapy in the management of advanced ovarian and primary peritoneal cancers. This response should not be surprising when one considers both the number of years primary surgical cytoreductive procedures have been the standard-of-care and the number of trainees worldwide who have been taught this should be the standard. 
In fact, some have argued (in the absence of any randomized trial data) for essentially the opposite approach in patients with the most advanced disease and have advocated for even more aggressive primary surgery (including resection of metastatic cancer in the liver and chest) rather than chemotherapy followed by surgery after a favorable response is documented [12]. The justification for this strategy is once again retrospective data demonstrating patients with the smallest volume of residual cancer (microscopic disease only) have the most favorable outcomes.

As previously noted, the problem with this conclusion is that it remains completely unknown if such outcomes are the result of the impressive skills of individual surgeons (and those of the institutions caring for patients undergoing such surgery) or simply the selection of patients with the most favorable tumor biology. Of course, it is possible that the true answer to this important question is a variable combination of both factors.

However, despite statements by some in the gynecologic oncology community to the contrary, the current evidence from the conduct of two well-designed and conducted phase 3 randomized trials strongly indicates that acceptable initial management of advanced ovarian cancer includes the delivery of neoadjuvant platinum-based chemotherapy (following the diagnosis of a malignancy consistent with ovarian cancer) and the subsequent performance of an interval cytoreductive procedure designed to remove all residual macroscopic cancer (if technically feasible). This surgery will then be followed by additional chemotherapy designed to optimize both the extent and duration of the response and overall survival.

\section{Author details}

Maurie Markman*

Address all correspondence to: maurie.markman@ctca-hope.com

From Cancer Treatment Centers of America, Eastern Regional Medical Center, Philadelphia, PA, USA

\section{References}

[1] Hennessy, B. T, Coleman, R. L, \& Markman, M. Ovarian Cancer (2009). , 374, 1371-1382.

[2] Markman, M. Concept of optimal surgical cytoreduction in advanced ovarian cancer: A brief critique and a call for action. J Clin Oncol (2007). , 25, 4168-4170.

[3] Griffin, T. Surgical resection of tumor bulk in the primary treatment of ovarian carcinoma. Natl Cancer Inst Monogr (1975). , 42, 101-104. 
[4] Bristow, R. E, Tomacruz, R. S, Armstrong, D. K, et al. Survival effect of maximal cytoreductive surgery for advanced ovarian carcinoma during the platinum era: A meta-analysis. J Clin Onocl (2002). , 20, 1248-1259.

[5] Van Der Burg, M. E, Van Lent, M, Buyse, M, et al. The effect of debulking surgery after induction chemotherapy on the prognosis in advanced epithelial ovarian cancer. N Engl J Med (1995). , 332, 629-634.

[6] Rose, P. G, Nerenstone, S, Brady, M. F, et al. Secondary surgical cytoreduction for advanced ovarian carcinoma. N Engl J Med (2004). , 351, 2489-2497.

[7] Bristow, R. E, \& Chi, D. S. Platinum-based neoadjuvant chemotherapy and interval surgical cytoreduction for advanced ovarian cancer: A meta-analysis. Gynecol Oncol (2006). , 103, 1070-1076.

[8] Schwartz, P. E, Rutherford, T. J, Chambers, J. T, et al. Neoadjuvant chemotherapy for advanced ovarian cancer: Long-term survival. Gynecologic Oncol (1999). , 72, 93-99.

[9] Surwit, E, Childers, J, Atlas, I, et al. Neoadjuavnt chemotherapy for advanced ovarian cancer. Int J Gynecol Cancer (1996). , 6, 356-361.

[10] Vergote I De Wever ITjalma W, et al. Neoadjuvant chemotherapy or primary debulking surgery in advanced ovarian carcinoma: A retrospective analysis of 285 patients. Gynecol Oncol (1998). , 71, 431-436.

[11] Vergote, I, Trope, C. G, Amant, F, et al. Neoadjuvant chemotherapy or primary surgery in stage IIIC or IV ovarian cancer. N Engl J Med (2010). , 363, 843-953.

[12] Chi, D. S, Eisenhauer, E. L, Zivanovic, O, et al. Improved progression-free and overall survival in advanced ovarian cancer as a result of a change in surgical paradigm. Gynecologic Oncol (2009). , 114, 26-31. 